\title{
A Case Report of Guillain-Barre Syndrome in an Eleven-Month Infant
}

\author{
Rossela Vega-Castro ${ }^{\mathrm{a}}$, Miguel Garcia-Dominguez ${ }^{\mathrm{b}, \mathrm{e}}$, Edgardo Tostado-Morales ${ }^{\mathrm{c}}$, \\ Giordano Perez-Gaxiola ${ }^{\mathrm{d}}$
}

\begin{abstract}
Guillain-Barre syndrome (GBS) is an acute immune-mediated progressive predominantly motor symmetric polyradiculoneuropathy which causes demyelination and leads to weakness, ataxia and areflexia. There are a variety of forms of the syndrome; and despite being the most common cause of acute flaccid paralysis in children, it has a low incidence under 18 years old, and it is even rarer in children less than 2 years of age. Very few cases have been reported under 12 months of age. We describe a case of an 11-month-old male infant presenting with weakness and inability to ambulate who was diagnosed with GBS.
\end{abstract}

Keywords: Flaccid paralysis; Guillain-Barre syndrome; Polyradiculoneuropathy

\section{Introduction}

Guillain-Barre syndrome (GBS) is an acute immune-mediated peripheral polyneuropathy with a variable clinical presentation. GBS has a low incidence of 0.5 - 2 cases per 100,000 children under 18 years old. It is rare in infants $[1,2]$. GBS is deemed to be an autoimmune disease, and infections caused by Campylobacter jejuni, cytomegalovirus (CMV), Epstein-Barr virus (EBV), influenza virus and Mycoplasma pneumoniae are reported as important triggers [3, 4]. The diagnosis of GBS is clinical and supported by neuroconductive studies, as well as

Manuscript submitted December 18, 2020, accepted December 28, 2020

Published online January 19, 2021

aDepartment of Neurology and Neurophysiology, Hospital Pediatrico de Sinaloa, Culiacan, Mexico

${ }^{b}$ Department of Clinical Immunology, Hospital Pediatrico de Sinaloa, Culiacan, Mexico

'Department of Emergency, Hospital Pediatrico de Sinaloa, Culiacan, Mexico ${ }^{\mathrm{d} E v i d e n c e-B a s e d ~ M e d i c i n e ~ D e p a r t m e n t, ~ H o s p i t a l ~ P e d i a t r i c o ~ d e ~ S i n a l o a, ~} \mathrm{Cu}-$ liacan, Mexico

${ }^{\mathrm{e} C o r r e s p o n d i n g ~ A u t h o r: ~ M i g u e l ~ G a r c i a-D o m i n g u e z, ~ D e p a r t m e n t ~ o f ~ C l i n i c a l ~}$ Immunology, Hospital Pediatrico de Sinaloa, Culiacan, Mexico.

Email: miguelgarcia.alergia@gmail.com

doi: https://doi.org/10.14740/jmc3638 the exclusion of differential diagnoses. Established treatment regimens include intravenous immunoglobulin (IVIG) and plasma exchange (PE) in older children and adults. However, efficacy and safety of these treatments have not been established in infants [4-9]. The prognosis for childhood GBS is often favorable [10].

\section{Case Report}

A previously healthy 11-month-old boy was referred to a pediatric hospital with a history of weakness and difficulty feeding for 10 days. He developed fever and diarrhea 2 weeks before the onset of weakness. He was treated with trimethoprim and sulfamethoxazole for 7 days. Two days after the diarrhea started, he presented weakness in the lower extremities and at 24 $\mathrm{h}$ in both arms, and later inability to sit, feed and support the head.

Pregnancy and delivery were unremarkable. He had achieved normal motor milestones. After 6 months of exclusive breastfeeding, solid foods were started. All vaccinations had been administered. He was vaccinated against diphtheria, tetanus, pertussis, polio, Hemophilus influenzae B, hepatitis B and rotavirus 4 months ago. He had no other health problems. The parents were healthy and not consanguineous. No family history of autoimmune diseases was reported.

On initial examination, he was in the 50th percentile for weight, length, and head circumference. The vital signs were in normal ranges according to his age. He was wide awake, making eye contact. On detailed neurological examination, pupils were isochoric and responsive to normal light. No abnormalities of cranial nerves were detected. He had flaccid paralysis with absence of inferior and superior deep tendon reflexes. There was no spontaneous movement in the lower extremities, whereas there was only proximal muscle contraction in the upper extremities. Plantar reflexes were absent. He responded to painful examination of the skin on the lower and upper extremities with grimaces. Other tests of the system were normal.

Laboratory studies that included complete blood count, biochemical parameters, and coagulation profile were normal. Examination of the cerebrospinal fluid revealed an increase in the protein count $(119 \mathrm{mg} / \mathrm{dL})$ and the cell count was in the normal range. Gram stain revealed no cells or microorganisms, and the cerebrospinal fluid culture was sterile. Stool culture was negative. Electroneuromyography revealed normal motor 
and sensory nerve conduction velocity, greatly reduced amplitude of action potentials, and an absence of $F$ responses consistent with acute motor axonal neuropathy (AMAN), a variant of GBS.

Patient received IVIG at $2 \mathrm{~g} / \mathrm{kg}$ single dose. Twenty-four hours later, evidence of proximal muscle contraction in upper and lower extremities, $72 \mathrm{~h}$ later horizontal movement of the lower extremities when gravity is eliminated. Two weeks later proximal movements against gravity overcome in upper and lower extremities, and he can hold his head. One month later he can grasp a toy but still has inability to stand alone.

\section{Discussion}

GBS is an acute immune-mediated peripheral polyneuropathy; it is the most common cause of flaccid paralysis in children, characterized by progressive, symmetrical weakness, paresthesia and areflexia, hyporeflexia or both [11].

It is more frequent in the adult population with an increase of $20 \%$ for every 10 years of increase in age after the first decade of life. In pediatric ages it is observed less frequently, and rarely in children under 2 years of age $[12,13]$. A high index of suspicion is required in children under 2 years of age considering differential diagnoses such as acute cerebellar ataxia, transverse myelitis, spinal cord compression, tick-borne paralysis, botulism, trauma, acute disseminated encephalomyelitis, myasthenia gravis, poliomyelitis, and additional myopathies [14].

There are cases reported in children under 12 months with neurophysiological findings compatible with peripheral polyneuropathy where the diagnosis of GBS was excluded. Royden et al reported three cases, a 16-day-old male with symptoms from birth in which it was not possible to differentiate between a congenital error of myelination vs. GBS; another 11-monthold male who had a history of SABIN application, 3 weeks later developed respiratory failure and depended on ventilation up to 2 years of age. The third case of a 4.5-month-old girl with feeding difficulties, generalized hypotonia with ventilatory dependence, was identified, where the presence of botulinum toxin type A was determined in both stool and honey [15].

In 1959 Merril et al reported the first case of an 8-monthold infant in a series of 37 patients with GBS [16], while Cantalini et al in 1960 reported a 2-month-old patient with GBS treated with dexamethasone, prior to the use of IVIG [17].

Anecdotal cases with ages under 12 months are reported in the literature of GBS, with great diversity in their clinical presentation, diagnostic approach, treatment and evolution. Table 1 shows GBS cases younger than 12 months [4, 18-21].

\section{Conclusions}

GBS is a rare disease, and individual studies often lack a sufficient number of cases to make age-specific incidence estimates. The symptoms, clinical course, and paraclinical findings in childhood GBS have rarely been investigated in non-gait children. GBS is an entity that is considered serious that requires multidisciplinary management to prevent complications from occurring and thereby improve the prognosis of patients. Upon arrival at the emergency department, all patients should be carefully evaluated, looking for signs of autonomic and respiratory dysfunction. It has been seen that the pediatric patient has a better prognosis compared to the adult. Approximately $80 \%$ of patients who have suffered GBS are symptomatic within a period of 6 months and $20 \%$ persist with a moderate neurological sequela. It has been proven that the initiation of treatment in the early stages of the disease produces a faster recovery and consequently fewer sequelae.

\section{Acknowledgments}

None to declare.

\section{Financial Disclosure}

None to declare.

\section{Conflict of Interest}

None to declare.

\section{Informed Consent}

Informed consent was given to use picture and clinical information.

\section{Author Contributions}

$\mathrm{RV}$ and MG were the treating physicians and the monitoring of the neurological disease. RV wrote the manuscript with the support of MG, ET and GP supervised the project. All authors discussed the results and contributed to the writing of the final manuscript.

\section{Data Availability}

Any inquiries regarding supporting data availability of this study should be directed to the corresponding author.

\section{References}

1. Agrawal S, Peake D, Whitehouse WP. Management of children with Guillain-Barre syndrome. Arch Dis Child Educ Pract Ed. 2007;92(6):161-168.

2. Winner SJ, Evans JG. Age-specific incidence of Guillain-Barre syndrome in Oxfordshire. Q J Med. 1990;77 (284):1297-1304.

3. Hughes RA, Cornblath DR. Guillain-Barre syndrome. Lancet. 2005;366(9497):1653-1666. 

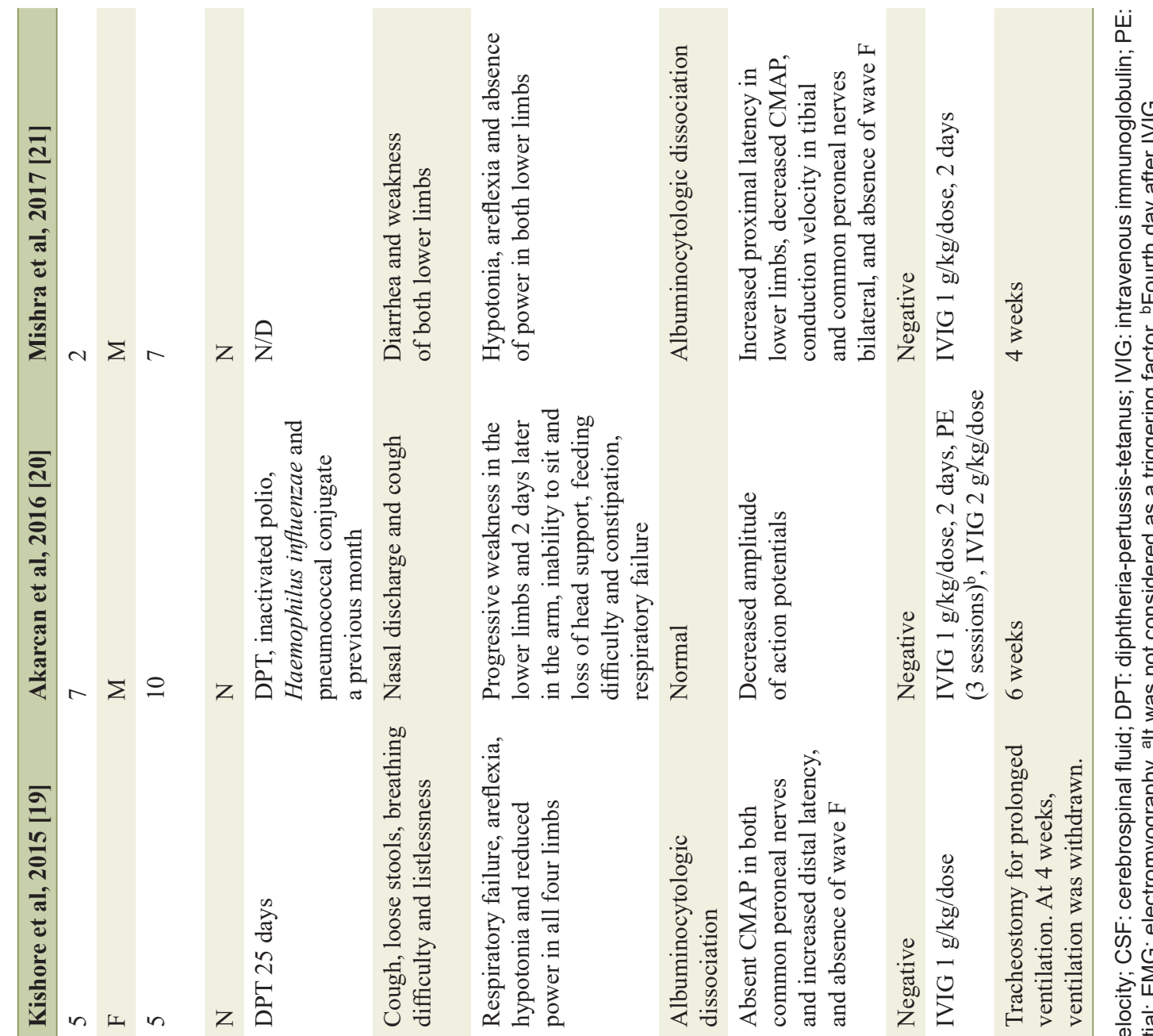

年
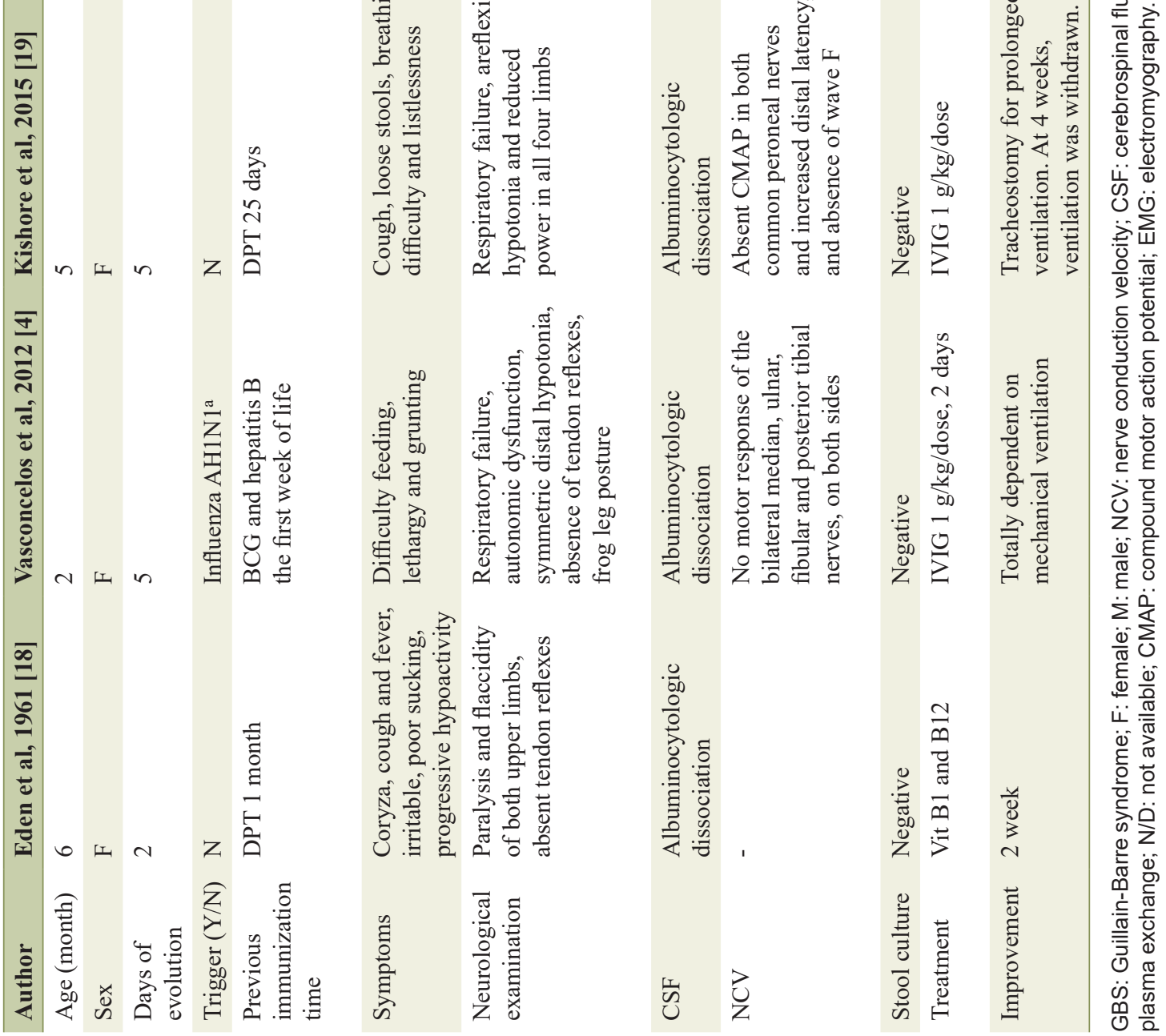
4. Vasconcelos A, Abecasis F, Monteiro R, Camilo C, Vieira M, de Carvalho M, Correia M. A 3-month-old baby with H1N1 and Guillain-Barre syndrome. BMJ Case Rep. 2012;2012:bcr1220115462.

5. Korinthenberg R, Schessl J, Kirschner J. Clinical presentation and course of childhood Guillain-Barre syndrome: a prospective multicentre study. Neuropediatrics. 2007;38(1):10-17.

6. Hughes RA, Raphael JC, Swan AV, Doorn PA. Intravenous immunoglobulin for Guillain-Barre syndrome. Cochrane Database Syst Rev. 2004;1:CD002063.

7. Hughes RA, Brassington R, Gunn AA, van Doorn PA. Corticosteroids for Guillain-Barre syndrome. Cochrane Database Syst Rev. 2016;10:CD001446.

8. Chevret S, Hughes RA, Annane D. Plasma exchange for Guillain-Barre syndrome. Cochrane Database Syst Rev. 2017;2:CD001798.

9. Hughes RA, Pritchard J, Hadden RD. Pharmacological treatment other than corticosteroids, intravenous immunoglobulin and plasma exchange for Guillain Barre syndrome. Cochrane Database Syst Rev. 2011;3:CD008630.

10. Rosen BA. Guillain-Barre syndrome. Pediatr Rev. 2012;33(4):164-170; quiz 170-161.

11. Willison HJ, Jacobs BC, van Doorn PA. Guillain-Barre syndrome. Lancet. 2016;388(10045):717-727.

12. Yuki N, Hartung HP. Guillain-Barre syndrome. N Engl J Med. 2012;366(24):2294-2304.
13. Sejvar JJ, Baughman AL, Wise M, Morgan OW. Population incidence of Guillain-Barre syndrome: a systematic review and meta-analysis. Neuroepidemiology. 2011;36(2):123-133.

14. Chung A, Deimling M. Guillain-Barre Syndrome. Pediatr Rev. 2018;39(1):53-54.

15. Jones HR, Jr. Guillain-Barre syndrome: perspectives with infants and children. Semin Pediatr Neurol. 2000;7(2):91102.

16. Merrill, RE y Fredrickson D. Sindrome de Landry-Guillain-Barre. The Journal of Pediatrics. 1959;54(6):816822.

17. Cantalini C. [Guillain-Barre polyradiculoneuritis in a 2-month-old infant treated with dexamethazone]. Minerva Pediatr. 1960;12:1322-1325.

18. Eden AN. Guillain-Barre syndrome in a six-month-old infant. Am J Dis Child. 1961;102:224-227.

19. Kishore P, Sharma PK, Saikia B, Khilnani P. GuillainBarre syndrome masquerading as acute respiratory failure in an infant. J Pediatr Neurosci. 2015;10(4):399-400.

20. Akarcan SE, Isguder R, Yilmaz U, Ayhan Y, Ceylan G, Agin H. Guillain-Barre syndrome in a 7-month-old boy successfully applied plasma exchange. Transfus Apher Sci. 2016;54(1):139-143.

21. Mishra S, Mondal D, Mahar J, Gunasekar A, Seth S. Guillain-Barre Syndrome (GBS) in a 2-month-old infant: a case report. Sch J App Med Sci. 2017;5(3B):844-845. 